\title{
Hereditary Red Blood Cell Enzymopathies
}

\begin{tabular}{cll|} 
Author(s) & \multicolumn{2}{l}{ (1) Tekin Aksu } \\
\cline { 2 - 3 } Affiliation(s) & $\begin{array}{l}\text { Hacettepe University Faculty of Medicine, Department of Pediatrics, Division of Hematology, Ankara, } \\
\text { Turkey }\end{array}$ & \\
\cline { 2 - 3 } $\begin{array}{c}\text { Article } \\
\text { Information }\end{array}$ & $\begin{array}{l}\text { Article Type: Invited Review } \\
\text { Article Group: Pediatric Hematology }\end{array}$ & $\begin{array}{l}\text { Received: } 15.03 .2021 \\
\text { Accepted: } 21.04 .2021 \\
\text { Available Online: } 30.04 .2021\end{array}$ \\
\hline
\end{tabular}

\section{Abstract}

Red cell metabolic disturbances result in hemolysis, which leads to a significant shortening of the erythrocyte life span. The most common enzyme deficiencies are glucose 6-phosphate dehydrogenase (G6PD) in the antioxidant pathway, pyruvate kinase in the anaerobic glycolysis pathway, and pyrimidine 5' nucleotidase (P5'N) in the nucleotide metabolism. While the $\mathrm{X}$ chromosome inherits G6PD and phosphoglycerate kinase deficiencies, other enzymopathies show autosomal recessive inheritance. Although the causes of hereditary hemolytic disorders are diverse, clinical, laboratory findings and complications overlap. A history of neonatal jaundice requiring phototherapy and exchange transfusion is quite usual. Mild to severe anemia may be accompanied with episodic or constant hemolysis associated with icterus, hyperbilirubinemia, growth retardation, gallstones, splenomegaly, and a variable degree of iron overload. Erythrocyte enzyme disorders should be suspected in patients with severe hemolytic episodes, or chronic hemolysis, after excluding hemoglobinopathies, membranopathies, and immune-mediated hemolysis.

Keywords: Glucose 6-phosphate dehydrogenase deficiency, pyruvate kinase deficiency, glucose phosphate isomerase deficiency, triosephosphate isomerase deficiency, pyrimidine 5'-nucleotidase deficiency

\section{Introduction}

Erythrocytes use two main biochemical pathways to maintain their physiological state. The Embden-Meyerhof anaerobic pathway is used for energy, and the hexose monophosphate (HMP) shunt pathway for reducing potential toxicities. With the energy gained, cell shape, elasticity, intracellular cation, and water content are preserved. Purine and pyrimidine nucleotides are obtained through nucleotide metabolism.
The most common enzyme deficiencies are glucose 6-phosphate dehydrogenase (G6PD) in the antioxidant pathway, pyruvate kinase $(\mathrm{PK})$ in the anaerobic glycolysis pathway, and pyrimidine 5 'nucleotidase $\left(\mathrm{P}^{\prime} \mathrm{N}\right)$ in the nucleotide metabolism. While the $X$ chromosome inherits G6PD and phosphoglycerate kinase deficiency, other enzymopathies show autosomal recessive inheritance. ${ }^{1}$

Correspondence: Tekin Aksu, Hacettepe University Faculty of Medicine, Department of Pediatrics,

Division of Hematology, Ankara, Turkey

E-mail: tekinaksu@gmail.com 
Red cell metabolic disturbances result in hemolysis, which leads to a significant shortening of the erythrocyte life span. Although the causes of hereditary hemolytic disorders are diverse, clinical, laboratory findings and complications overlap. A history of neonatal jaundice requiring phototherapy and exchange transfusion is quite usual. Mild to severe anemia may be associated with episodic or constant hemolysis associated with icterus, hyperbilirubinemia, growth retardation, gallstones, splenomegaly, and a variable degree of iron overload. Reticulocytosis due to compensatory erythropoiesis usually accompanies with the disease, except in some forms of PK deficiency cases

with ineffective erythropoiesis. Erythrocyte enzyme disorders should be suspected in patients with severe episodic or chronic hemolytic anemia, after excluding hemoglobinopathies, membranopathies, and immunemediated hemolysis. These patients may experience accelerated hemolysis with infections or aplastic crises due to parvovirus infection.

\section{Glucose-6-phosphate dehydrogenase deficiency}

Glucose-6-phosphate dehydrogenase deficiency is the most common enzyme deficiency in the HMP pathway that causes hemolytic anemia. G6PD is involved in the production of nicotinamide adenine dinucleotide phosphate (NADPH), which protects the cell from oxidant injury triggered by certain drugs or infections by ensuring a reduced state of glutathione $(\mathrm{GSH}){ }^{2}$ Its deficiency mostly affects erythrocytes. Erythrocytes do not have alternative pathways for NADPH production. In NADPH deficiency, hemolysis occurs by precipitation of hemoglobin (Heinz body) in erythrocytes by oxidative stress and damaging the erythrocyte membrane. ${ }^{2}$ G6PD deficiency leads to episodic or chronic non-spherocytic hemolytic anemia. It affects more than 400 million people around the world. ${ }^{3}$ Its prevalence in the world is $4.9 \%{ }^{3}$ While it is common in males due to its X-linked inheritance (hemizygous), in populations where mutations are common, the disease due to homozygous mutations is reported in females. Besides, in carrier womens, moderate-to-mild findings due to $\mathrm{X}$ chromosome inactivation (Lyon hypothesis) can be seen. ${ }^{2}$ G6PD deficiency is common in Africa, Asia, the Mediterranean, and the Middle East regions. The disease is more common in areas where malaria was once endemic due to the protection of G6PD deficiency against Plasmodium falciparum malaria. ${ }^{4}$ The frequency of the G6PD deficiency was reported to vary from 0.5 $2.9 \%$ in Turkey. ${ }^{5}$ It has been reported with a rate of $8.2 \%$ in the Çukurova region. ${ }^{5}$ The World Health Organization classified G6PD deficiency according to enzyme activity and the degree of hemolysis (Table 1). ${ }^{6}$ Of note, the normal enzyme is referred to as G6PD B (wild-type).

Normally, the G6PD activity declines with red blood cell aging. In mild G6PD variants (Class III), especially older erythrocytes are affected due to declining enzymatic activity. Whereas in the variants of the Class II, including the Mediterranean variant, erythrocytes of all ages are deficient in the enzyme. Class II variants are more susceptible to oxidant damage and may experience severe hemolysis.

\section{ighlights}

- Glucose 6-phosphate dehydrogenase often associated with episodic hemolysis triggered by deficiency should tients with chronic subtle peripheral after excluding ies, membranopathies, ed hemolysis.

\section{Clinical findings}

The patients with most G6PD variants are usually asymptomatic, and there are no associated laboratory findings related to hemolysis. The acute hemolytic episode may pursue oxidant injury within 2448 hours, triggered by certain drugs, toxins, infections, and fava beans. It seems infections are the most common cause of hemolytic episodes, especially in children. ${ }^{1}$ Jaundice, pallor, dark urine develop, and mild to severe hemoglobin decrease may be seen after exposure. Drugs and chemicals that cause hemolysis and should be avoided are listed elsewhere. , 2,7 While some drugs do not cause clinically significant hemolysis in therapeutic doses, such as low-dose aspirin and trimethoprim-sulfamethoxazole, high doses are associated with hemolysis. An acute hemolytic process is seen due to fava beans consumption, which is called favism, particularly in Mediterranean and Asian G6PD variants. Fava contains divicine, isouramil, and convicine, which increase reactive oxygen products and induce hemolysis.

In the neonatal period, it may cause spontaneous hemolysis and hyperbilirubinemia, even kernicterus. ${ }^{8}$ In most cases, jaundice is much more common than anemia. If the patient has Gilbert's syndrome [uridinediphosphate-glucuronyl transferase 1 family, polypeptide A1 (UGT1A1) mutation], the severity of hemolysis increases. Neonates with severe jaundice should be tested for G6PD deficiency. Exposure of the neonate and mother to drugs that cause oxidant stress, acidosis, and hypoxia may trigger hemolysis.

Table 1

The World Health Organization classification of G6PD deficiency

\section{Class I: Severe deficiency}

Chronic non-spherocytic hemolytic anemia

Sporadic and rare

Residual enzyme activity: $<10 \%$ of normal

Class II: Severe deficiency

Episodic severe hemolysis with oxidant injury Mediterranean or Asian variants

Residual enzyme activity: $<10 \%$ of normal

Class III: Mild to moderate deficiency

Intermittent acute hemolysis with oxidant injury

Common A- variant

African American

Residual enzyme activity: $10-60 \%$ of normal

Class IV: Normal activity

Enzyme activity: $60-150 \%$ of normal

No clinical findings

Class V: Increased enzyme activity

Enzyme activity: $>150 \%$ of normal

No clinical findings 
In rare instances, G6PD Class I deficient patients have chronic nonspherocytic hemolytic anemia that indistinguishable from other glycolytic enzyme disorders. They have mild to moderate anemia with a high reticulocyte count between $10-15 \%$.

\section{Laboratory findings}

With acute hemolytic episodes, patients with G6PD deficiency present with sudden drops in hemoglobin and hematocrit. Anemia is normochromic, normocytic, accompanied by reticulocytosis within five days of onset of a hemolytic episode. Polychromasia, anisocytosis, poikilocytosis, spherocytes, bite, or blister-shaped erythrocytes are seen in the peripheral blood smear. Hemolysis occurs both intravascular or extravascular. Oxidized or denatured hemoglobin deposits called Heinz bodies can be seen in erythrocytes with supravital stains, where these deposits will be cleared from circulation in 3 to 4 days. Hemoglobinuria, an increase in indirect bilirubin, free hemoglobin, and lactate dehydrogenase (LDH), decreased haptoglobin are other accompanying findings.

G6PD enzyme activity can be measured quantitatively by a spectrophotometric assay. However, normal or high enzyme levels due to reticulocytosis can be seen during the hemolytic episode, which leads to falsenegative results. Therefore, in suspected patients, the enzyme activity should be reevaluated three months after the hemolytic episode when all ages of erythrocytes repopulated. Furthermore, in patients who have received erythrocyte transfusion in the last three months, the erythrocyte enzyme levels may be found to be normal, even if there is a deficiency. Moreover, molecular studies have shown more than 160 different gene mutations in G6PD deficiency. ${ }^{9}$ These studies identified that almost all mutations are missense. Gene sequencing is not necessary for patients with common phenotypes, such as patients with episodic hemolysis. However, these studies help confirm the diagnosis of Class I G6PD deficiency.

\section{Prevention and Treatment}

The most important approach is to secure from hemolytic episodes. Family and patient should be educated about the hemolytic episode findings such as dark urine, jaundice, and weakness. Besides, they shouldn't consume foods containing fava beans and should avoid drugs with known effects of oxidant damage. When a hemolytic episode occurs, depending on the degree of anemia, transfusion may be required. Erythrocyte suspension should be given to patients whose hemoglobin level is below $7 \mathrm{~g} / \mathrm{dl}$ or $9 \mathrm{~g} / \mathrm{dl}$ and whose hemoglobinuria continues or is symptomatic with rapid hemoglobin decrease. Patients who do not need transfusion should be followed for at least 48 hours. Severe hemolysis can lead to acute renal failure, and supportive treatment may be required.

In patients with chronic anemia, the hemoglobin level should be kept between 8-10 g/dl and followed up for iron overload. Splenectomy can also be performed in patients with severe chronic anemia and who have symptoms of hypersplenism.

\section{Disorders of the glycolytic enzymes}

G6PD deficiency is far more common than glycolytic enzyme abnormalities. While the most common cause of glycolytic pathway disorders is PK deficiency, diseases related to deficiencies of all other enzymes have also been described. Glycolytic pathway enzymopathies usually show an autosomal recessive inheritance.

\section{Pyruvate Kinase Deficiency}

Hemolyticanemias resulting from the red cell metabolism's defects lack specific morphologic abnormalities on the peripheral blood smear since they are called hereditary nonspherocytic hemolytic anemias (HNSHA). Valentine et al. discovered that PK leads to HNSHA. ${ }^{10}$ To date, over 600 families with PK deficiency have been reported, which is the most common glycolytic enzyme disorder that leads to HNSHA. ${ }^{11,12}$ Its prevalence is reported as 51 per million. ${ }^{13,14}$ The rarity of PK deficiency and the variable clinical findings cause difficulties in diagnosis, often underdiagnosed. The other factors contributing to underdiagnosis are regular transfusions interfering with diagnostic tests and PK enzyme measurements which are not standard between institutions. PK deficiency is caused by compound heterozygote or homozygote mutations in the gene PKLR. ${ }^{11-13}$ Drastic mutations lead to a total loss of PK activity accompanied with a more severe disease such as intrauterine death or severe neonatal anemia. If PK enzyme activity is preserved at some point in different mutations, leading to milder phenotypic consequences. Mostly missense (70-80\%), splicing, and premature stop codon mutations have been identified in the PKLR gene. ${ }^{15,16}$

The glycolytic pathway maintains ATP, 2,3-diphosphoglycerate (2,3-DPG), and $\mathrm{NADH}$ to modulate hemoglobin oxygen affinity and reduce methemoglobin. ${ }^{17} \mathrm{PK}$ catalyzes phosphoenolpyruvate's conversion to pyruvate in the glycolytic pathway and generates $50 \%$ of erythrocyte ATP production. ${ }^{18}$ As a result of PK deficiency, ATP decreases, while intermediate metabolites, including 2,3-DPG, increase. ATP depletion results in cation loss, dehydration, and red cell damage that eventually clearance in the spleen. ${ }^{19,20}$

\section{Hematological Features}

The hematological features of PK deficiency, including increased reticulocyte count, reduced haptoglobin, and elevated bilirubin, overlaps with other hereditary hemolytic anemias. ${ }^{1}$ Patients with PK deficiency may present with mild to severe anemia and reticulocytosis. ${ }^{12}$ Red blood cell morphology is usually unremarkable, except for spiculated erythrocytes. Also, these spiculated erythrocytes increase after splenectomy. An increase of 2,3 DPG balances tissue oxygen delivery and mitigates symptoms of anemia. PK deficiency causes extravascular hemolysis where a significant increase in $\mathrm{LDH}$ is not expected. ${ }^{17,21}$ The osmotic fragility test is normal, but the autohemolysis test is usually abnormal. Recently rare PK deficiency cases associated with inappropriately low reticulocytes and dyserythropoietic features at bone marrow examination are defined. These cases reflect ineffective erythropoiesis due to PK deficiency, resulting in misdiagnosis with CDAs. ${ }^{17}$ 


\section{Clinical Features}

Anemia-related symptoms, splenomegaly, and jaundice are common in patients with PK deficiency. ${ }^{12,17,22,23}$ There are variable perinatal complications including, fetal anemia, nonimmune hydrops, and neonatal hyperbilirubinemia requiring phototherapy or exchange transfusion. ${ }^{12}$ Even cases that could progress to liver failure in the neonatal period were reported. ${ }^{24}$ Severe anemia due to chronic hemolysis may pursue until splenectomy in childhood. ${ }^{12}$ There are also mild cases with a compensated anemia that may not be noticed until adult age. In adults, anemia is relatively stable, though acute worsening of anemia may occur during infections, aplastic crises, pregnancy, or increased hemolysis. Patients with biallelic null or nonmissense mutations present with lower hemoglobin values and more transfusion need, high risk of complications, while they show inadequate response to splenectomy. ${ }^{12,25}$

\section{Diagnosis}

There is no simple screening test available for enzymopathies, and particularly it is more challenging in transfusion-dependent patients. Other hemolytic anemias, including immune-mediated hemolysis, hemoglobinopathies, or membranopathies, should be excluded. ${ }^{11,17}$ Molecular studies or a low level of PK enzyme activity with a spectrophotometric assay can be diagnostic. ${ }^{26}$ PK enzyme activity is confounded by increased reticulocytes, recent transfusions, and contaminating leukocytes with high specific enzyme activity. Moreover, the abnormal kinetics of the enzyme in vivo may not reflect in vitro results. Furthermore, the reduced activity of the PK enzyme does not correlate with the severity of the disease. If the PK enzyme level is close to normal or normal, but the other erythrocyte enzyme levels are high, particularly hexokinase (HK) and

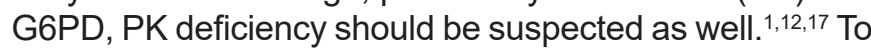
exclude this issue, it is recommended to measure $\mathrm{PK} / \mathrm{HK}$ ratio or $\mathrm{PK} / \mathrm{G} 6 \mathrm{PD}$ ratio. ${ }^{1}$

In transfusion-dependent patients, a survey reported various centers recommending 40-120 days to be spared after the last transfusion to perform an enzyme activity. That report suggests 50 days after transfusion may result in $6-12 \%$ contamination of donor erythrocytes and will not lead to a missed diagnosis. ${ }^{11}$ Recent reports suggest two diagnostic algorithms for PK deficiency. The first approach recommends measuring PK enzyme activity then confirming the diagnosis by molecular studies. The second approach is to screen disease by next-generation sequencing (NGS) targeted panels and confirm the diagnosis by measuring enzyme activity. ${ }^{11,17}$ Currently, most of the patients are screening by NGS panels targeted for hereditary hemolytic anemias, including PKLR.

\section{Therapy}

PK deficiency patients are currently managed with supportive treatments such as erythrocyte transfusion, splenectomy, folic acid supplementation, and iron chelation. ${ }^{17}$ The need for transfusion is higher in young children. The decision for transfusion should be made according to the hemoglobin level and by paying attention to the patient's tolerance of anemia and the patient's growth and development. ${ }^{17}$ Splenectomy results in reticulocytosis, increased hemoglobin, decreased or even eliminating transfusion requirements, and decreased bilirubin level in patients with PK deficiency. ${ }^{12}$ Unfortunately, splenectomy increases the risk of sepsis, thromboembolic events, and pulmonary hypertension. ${ }^{12,17}$ Currently, mitapivat (AG-348) is an allosteric activator of erythrocyte PK enzyme used in clinical trials in PK deficiency patients. ${ }^{27,28}$ Hematopoietic stem cell transplantation is an investigational treatment for PK deficiency patients and should not be recommended routinely. $^{29}$

Iron overload may develop as high as $48 \%$ of the patients with PK deficiency due to transfusion-related or ineffective erythropoiesis, particularly in adults. ${ }^{12}$ Besides, patients should be followed up regularly for the development of gallstones. Aplastic crises, leg ulcers, biliary tract disease, and extramedullary hematopoiesis may occasionally develop. ${ }^{12}$

\section{Glucose Phosphate Isomerase Deficiency}

Glucose phosphate isomerase (GPI) deficiency is the second most common glycolytic enzymatic defect, shows autosomal recessive inheritance. ${ }^{1}$ GPI deficiency associated with HNSHA of variable severity. ${ }^{30}$ Its approximate fraction constitutes 3-5\% of all enzymopathies. ${ }^{1}$ For diagnosis, measurement of this specific enzyme assay and molecular studies can be done. Clinical symptoms and complications of GPI deficiency overlap with PK deficiency. However, neuromuscular impairment such as hypotonia, ataxia, dysarthria, mental retardation has also been described in patients with GPI deficiency. ${ }^{30}$ Splenectomy reduces the need for erythrocyte transfusion. ${ }^{31}$

In addition to PK and GPI deficiency, hexokinase, phosphofructokinase, aldolase, triosephosphate isomerase, phosphoglycerate kinase, adenosine deaminase excess, and adenylate kinase deficiencies are reported even rarer. These enzymopathies may present with mild to severe HNSHA and neurologic deficits, and myopathy. 1

\section{Triosephosphate isomerase deficiency}

Triosephosphate isomerase (TPI) deficiency is a rare disease of the glycolytic pathway, shows autosomal recessive inheritance. TPI ensures the equilibration of the triosephosphates in the glycolytic pathway, wherein connection with lipid metabolism, glycerol-3-phosphate shuttle, and pentose phosphate pathway. ${ }^{32}$ TPI deficiency does not affect the erythrocyte's energy metabolism; however, it leads to toxic accumulation of methylglyoxal. Progressive neurologic impairment, cardiomyopathy, and increased susceptibility to infections accompany hemolytic anemia, which is frequently fatal in early childhood. ${ }^{32}$ No effective therapy is available for TPI deficiency. Other treatments are blood transfusions to treat anemia during hemolytic episodes and assisted ventilation to treat paralysis of the diaphragm. Hematopoietic stem cell transplantation was attempted in a few patients, but the outcome was poor.

\section{Hereditary pyrimidine 5'-nucleotidase deficiency}

Pyrimidine 5'-nucleotidase deficiency ( $\left.5^{\prime} \mathrm{N}\right)$, which is the most common enzyme deficiency in erythrocyte nucleotide metabolism, shows autosomal recessive inheritance; patients have less than 10\% normal P5'N 
activity. The prevalence of P5' $\mathrm{N}$ deficiency is not known. It occurs as a result of biallelic mutations in the NT5C3A gene on chromosome 7.33 There is no clinical finding in heterozygous mutations. It causes hereditary nonspherocytic hemolytic anemia. P5'N provides catalysis of pyrimidine nucleotides. In P5'N deficiency, pyrimidine nucleotides accumulate in erythrocytes and cause basophilic punctuation in erythrocytes. ${ }^{1}$ The mechanisms that cause erythrocyte destruction in this disease are not fully known. The median age at which the patients are diagnosed is 15 years ( 3 months-64 years). ${ }^{33}$

Symptoms overlap as other chronic hemolytic anemias such as jaundice, gallstones, and splenomegaly. ${ }^{1}$ Patients receiving regular transfusions due to severe anemia are rare. In most of the patients, mild to moderate anemia have been reported. The median hemoglobin value was $9.5 \mathrm{~g} /$ $\mathrm{dl}(2.8-15.2 \mathrm{~g} / \mathrm{dl}) .{ }^{33}$ In addition to the laboratory findings of reticulocytosis and hemolytic anemia, the basophilic punctuation on the erythrocytes helps diagnose, but it is not specific. The demonstration that the accumulation of pyrimidine nucleotides and decreased P5' $\mathrm{N}$ activity in erythrocytes is diagnostic. There is no specific treatment for the disease, and supportive treatments are applied. Splenectomy usually does not stop hemolysis. Patients should be monitored for iron overload which can also be seen in non-transfusion-dependent patients.

Conflict of Interest Statement: The authors have no conflicts of interest to declare.

Financial Disclosure: The authors declared that this study has received no financial support.

Peer-review: Externally peer-reviewed.

\section{References}

1. Grace RF, Glader B. Red Blood Cell Enzyme Disorders. Pediatr Clin North Am. 2018;65:579-595. [CrossRef]

2. Beutler E. Glucose-6-phosphate dehydrogenase deficiency: a historical perspective. Blood. 2008;111:16-24. [CrossRef]

3. Nkhoma ET, Poole C, Vannappagari V, Hall SA, Beutler E. The global prevalence of glucose-6-phosphate dehydrogenase deficiency: a systematic review and meta-analysis. Blood Cells Mol Dis. 2009;42:267-278. [CrossRef]

4. Nagel RL, Roth EF Jr. Malaria and red cell genetic defects. Blood. 1989;74:1213-1221. [CrossRef]

5. Altay Ç, Gümrük F. Red cell glucose-6-phosphate dehydrogenase deficiency in Turkey. Türkiye'de eritrosit glukoz-6-fosfat dehidrogenaz (G6PD) enzim eksikliği. Turk J Haematol. 2008;25:1 7. (In Turkish) [CrossRef]

6. Beutler E. The molecular biology of enzymes of erythrocyte metabolism. The Molecular Basis of Blood Disease. 1993; 29.

7. Youngster I, Arcavi L, Schechmaster R, et al. Medications and glucose-6-phosphate dehydrogenase deficiency: an evidencebased review. Drug Saf. 2010;33:713-726. [CrossRef]

8. Johnson L, Bhutani VK, Karp K, Sivieri EM, Shapiro SM. Clinical report from the pilot USA Kernicterus Registry (1992 to 2004). J Perinatol. 2009;29:S25-S45. [CrossRef]

9. Mason PJ, Bautista JM, Gilsanz F. G6PD deficiency: the genotypephenotype association. Blood Rev. 2007;21:267-283. [CrossRef]

10. Valentine WN, Tanaka KR, Miwa S. A specific erythrocyte glycolytic enzyme defect (pyruvate kinase) in three subjects with congenital non-spherocytic hemolytic anemia. Trans Assoc Am Physicians. 1961; 74:100-110. [CrossRef]
11. Bianchi $P$, Fermo E, Glader B, et al. Addressing the diagnostic gaps in pyruvate kinase deficiency: Consensus recommendations on the diagnosis of pyruvate kinase deficiency. Am J Hematol. 2019;94:149-161. [CrossRef]

12. Grace RF, Bianchi $P$, van Beers EJ, et al. Clinical spectrum of pyruvate kinase deficiency: data from the Pyruvate Kinase Deficiency Natural History Study. Blood. 2018;131:2183-2192. [CrossRef]

13. Secrest MH, Storm M, Carrington C, et al. Prevalence of pyruvate kinase deficiency: A systematic literature review. Eur J Haematol. 2020;105:173-184. [CrossRef]

14. Beutler E, Gelbart T. Estimating the prevalence of pyruvate kinase deficiency from the gene frequency in the general white population. Blood. 2000; 95:3585-3588. [CrossRef]

15. Canu G, De Bonis M, Minucci A, Capoluongo E. Red blood cell PK deficiency: An update of PK-LR gene mutation database. Blood Cells Mol Dis. 2016;57:100-109. [CrossRef]

16. Zanella A, Fermo E, Bianchi P, Chiarelli LR, Valentini G. Pyruvate kinase deficiency: the genotype-phenotype association. Blood Rev. 2007;21:217-231. [CrossRef]

17. Grace RF, Mark Layton D, Barcellini W. How we manage patients with pyruvate kinase deficiency. Br J Haematol. 2019;184:721734. [CrossRef]

18. Grace RF, Zanella A, Neufeld EJ, et al. Erythrocyte pyruvate kinase deficiency: 2015 status report. Am J Hematol. 2015;90:825-830. [CrossRef]

19. Nathan DG, Oski FA, Miller DR, Gardner FH. Life-span and organ sequestration of the red cells in pyruvate kinase deficiency. $N$ Engl J Med. 1968;278:73-81. [CrossRef]

20. Mentzer WC Jr, Baehner RL, Schmidt-Schönbein H, Robinson $\mathrm{SH}$, Nathan DG. Selective reticulocyte destruction in erythrocyte pyruvate kinase deficiency. J Clin Invest. 1971;50:688-699. [CrossRef]

21. Barcellini W, Fattizzo B. Clinical Applications of Hemolytic Markers in the Differential Diagnosis and Management of Hemolytic Anemia. Dis Markers. 2015;2015:635670. [CrossRef]

22. Zanella A, Bianchi P. Red cell pyruvate kinase deficiency: from genetics to clinical manifestations. Baillieres Best Pract Res Clin Haematol. 2000;13:57-81. [CrossRef]

23. Zanella A, Fermo E, Bianchi P, Valentini G. Red cell pyruvate kinase deficiency: molecular and clinical aspects. Br J Haematol. 2005;130:11-25. [CrossRef]

24. Raphaël MF, Van Wijk R, Schweizer JJ, et al. Pyruvate kinase deficiency associated with severe liver dysfunction in the newborn. Am J Hematol. 2007;82:1025-1028. [CrossRef]

25. Bianchi P, Fermo E, Lezon-Geyda K, et al. Genotype-phenotype correlation and molecular heterogeneity in pyruvate kinase deficiency. Am J Hematol. 2020;95:472-482. [CrossRef]

26. Hirono A, Forman L, Beutler E. Enzymatic diagnosis in nonspherocytic hemolytic anemia. Medicine (Baltimore). 1988;67:110117. [CrossRef]

27. Grace RF, Rose C, Layton DM, et al. Safety and Efficacy of Mitapivat in Pyruvate Kinase Deficiency. $N$ Engl $J$ Med. 2019;381:933-944. [CrossRef]

28. Rab MAE, Van Oirschot BA, Kosinski PA, et al. AG-348 (Mitapivat), an allosteric activator of red blood cell pyruvate kinase, increases enzymatic activity, protein stability, and ATP levels over a broad range of PKLR genotypes. Haematologica. 2021;106:238-249. [CrossRef]

29. van Straaten S, Bierings M, Bianchi P, et al. Worldwide study of hematopoietic allogeneic stem cell transplantation in pyruvate kinase deficiency. Haematologica. 2018;103:e82-e86. [CrossRef]

30. Kugler W, Lakomek M. Glucose-6-phosphate isomerase deficiency. Baillieres Best Pract Res Clin Haematol. 2000;13:89101. [CrossRef]

31. Iolascon A, Andolfo I, Barcellini W, et al. Recommendations regarding splenectomy in hereditary hemolytic anemias. Haematologica. 2017;102:1304-1313. [CrossRef]

32. Orosz F, Oláh J, Ovádi J. Triosephosphate isomerase deficiency: new insights into an enigmatic disease. Biochim Biophys Acta. 2009;1792:1168-1174. [CrossRef]

33. Zanella A, Bianchi P, Fermo E, Valentini G. Hereditary pyrimidine 5'-nucleotidase deficiency: from genetics to clinical manifestations. Br J Haematol. 2006;133:113-123. [CrossRef] 